\title{
Dysphagia: An unusual presentation of giant aneurysm of the right coronary artery and supravalvular aortic stenosis in Williams syndrome
}

\author{
Carmelo Mignosa, MD, FETCS, ${ }^{a}$ Salvatore Agati, MD, ${ }^{\text {a }}$ Salvatore Di Stefano, MD, ${ }^{a}$ \\ Giovanni Pizzimenti, MD, ${ }^{\mathrm{b}}$ Enrico Di Maggio, MD, ${ }^{\mathrm{c}}$ Dario Salvo, MD, ${ }^{\mathrm{d}}$ and \\ Giuseppe Ciccarello, FEBCP, ${ }^{\mathrm{e}}$ Messina and Milan, Italy
}

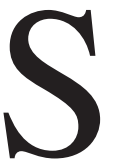

upravalvular aortic stenosis (SVAS) is typically associated with Williams syndrome. We describe the case of a mentally retarded 56-year-old man with Williams syndrome in whom giant aneurysm of the right coronary artery was an incidental finding during investigations for dysphagia.

\section{Clinical Summary}

A 56-year-old patient with dysphagia was admitted to our hospital for evaluation. On medical history, he was found to have neurodevelopmental delay and was receiving antihypertensive therapy. An extrinsic stenosis on the midportion of the esophagus did not allow us to complete full gastroenteral screening. Computed tomographic scanning showed the presence of a mediastinal mass 11 $\mathrm{cm}$ in diameter compressing the esophagus (Figure 1), which was diagnosed as an isolated aneurysm of the right sinus of Valsalva. The echocardiogram showed the presence of an SVAS with a gradient of $70 \mathrm{~mm} \mathrm{Hg}$, and the pulmonary trunk and bifurcation were normal. Angiography showed normal peripheral pulmonary vascularization and normal left coronary artery anatomy. The right coronary artery at its origin from the aorta opened up in a huge dilatation of $11 \mathrm{~cm}$. The patient was taken to the operating theater on an urgent basis. Cardiopulmonary bypass was instituted through femorofemoral cannulation. The heart was emptied, and the chest was opened. At opening, it was possible to visualize a huge aneurysmal mass compressing the right atrium and superior vena cava and covering the ascending aorta (Figure 2, A). The aorta was crossclamped and transected. Warm blood cardioplegic solution was administered directly into the coronary ostia. When the aorta

\footnotetext{
From the Pediatric Cardiac Surgery Unit, ${ }^{\mathrm{a}}$ Azienda U.S.L. 5, Ospedale San Vincenzo, Taormina (Messina), Italy; the Cardiology Unit, ${ }^{\mathrm{b}}$ Azienda U.S.L. 5, Ospedale di Milazzo, Messina, Italy; the Radiology Department, Azienda U.S.L. 5, Ospedale San Vincenzo, Taormina (Messina), Italy; the Pediatric Cardiac Intensive Care Unit, ${ }^{d}$ Azienda U.S.L. 5, Ospedale San Vincenzo, Taormina (Messina), Italy; and Perfusion Service Edwards Lifesciences, ${ }^{\text {e }}$ Milan, Italy.

Received for publication April 16, 2004; accepted for publication May 13, 2004.

Address for reprints: Carmelo Mignosa MD, FETCS, Unità Operativa di Cardiochirurgia, Presidio Ospedaliero "San Vincenzo," Contrada Sirina, 98039 Taormina (Messina), Italy (E-mail: carmignosa@tiscali.it).

J Thorac Cardiovasc Surg 2004;128:946-8

$0022-5223 / \$ 30.00$

Copyright (C) 2004 by The American Association for Thoracic Surgery doi:10.1016/j.jtcvs.2004.05.008
}

was opened, it was possible to visualize a tight SVAS (Figure $2, B$ ). The right coronary artery opened up in the aneurysm just from its origin. It was opened longitudinally. When the coronary artery had normal size, it was removed together with a cuff of aneu-

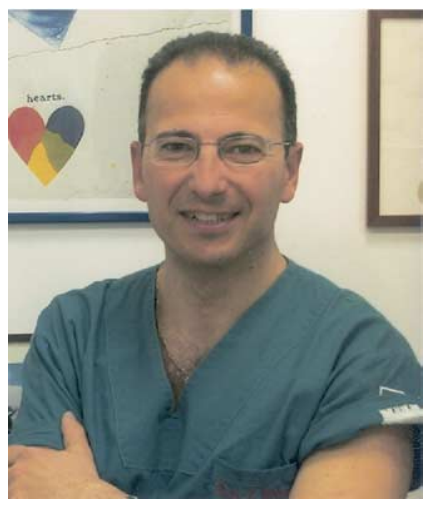

Dr Mignosa rysmal wall (Figure 2, $C$ ) and reimplanted directly onto the ascending aorta with a running 6-0 Prolene suture (Ethicon, Inc, Somerville, NJ). Three polytetrafluoroethylene (Gore-Tex; W. L. Gore \& Associates, Inc, Flagstaff, Ariz) patches were implanted with running 5-0 Prolene sutures to treat the SVAS (Figure 2, D).

The patient made an uneventful recovery and was discharged on postoperative day 6. Complete chromosomal evaluation confirmed the clinical suspicion of Williams syndrome. At 1 year's follow-up, he is free of symptoms, with normal electrocardiographic and echocardiographic findings.

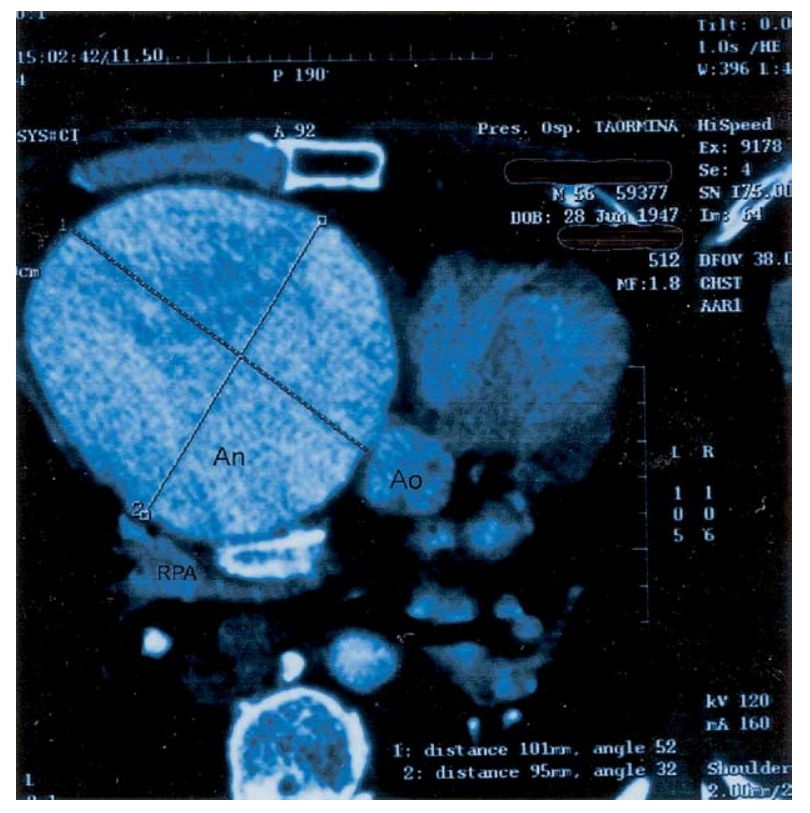

Figure 1. Computed tomographic scan. Ao, Aorta; An, right coronary artery aneurysm; $\boldsymbol{R P A}$, right pulmonary artery. 

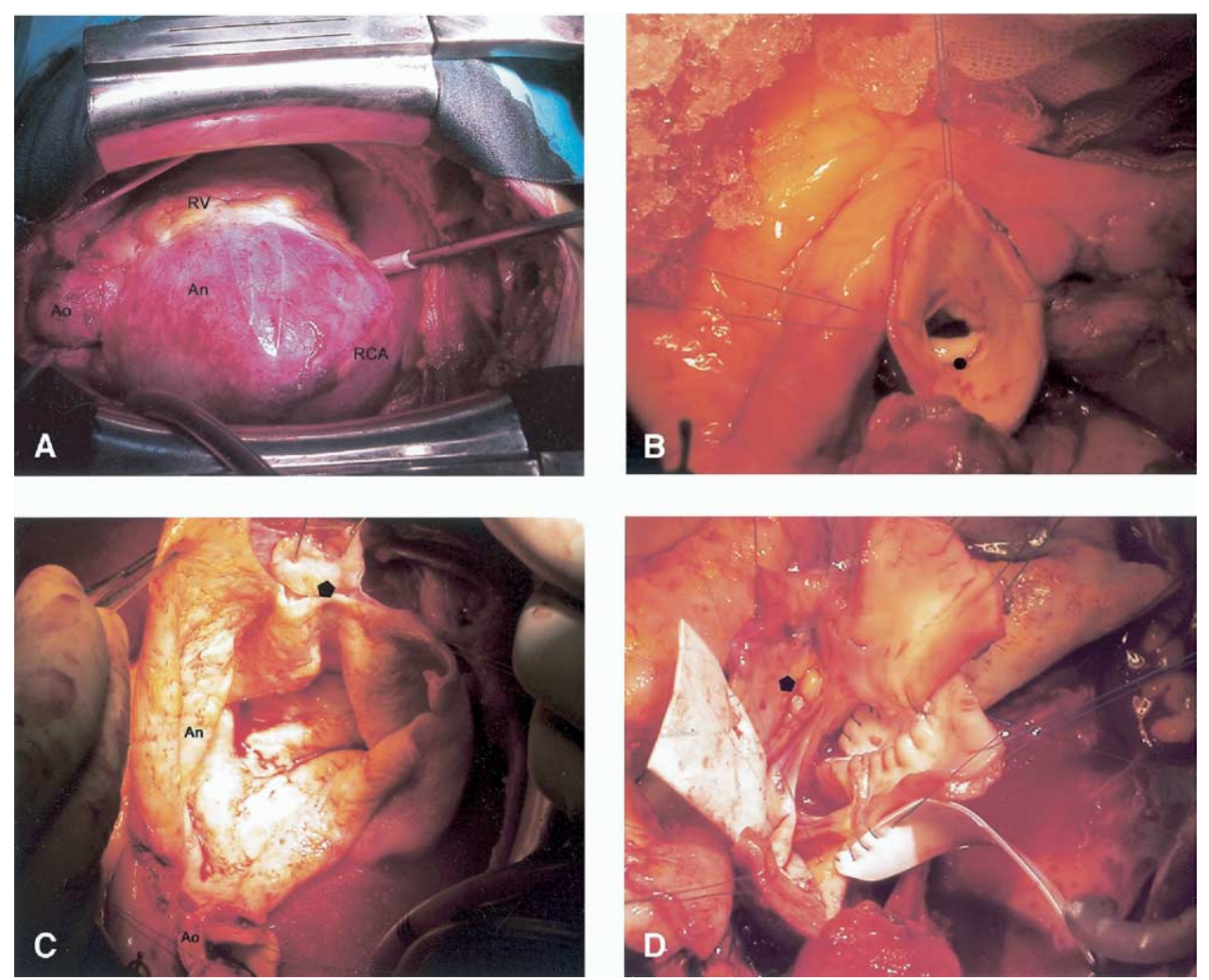

Figure 2. Operative findings. A, Aneurysm visualization at pericardial opening. B, SVAS. C, Aneurysmal sac opening with right coronary artery button incorporating 2 coronary ostia (probes). D, Multiple sinus reconstruction and direct right coronary artery reimplantation. Ao, Aorta; RCA, right coronary artery; An, right coronary artery aneurysm; RV, right ventricle; black circle, SAVS; black pentagon, right coronary artery button incorporating 2 coronary ostia.

\section{Discussion}

Williams syndrome is a contiguous gene syndrome associated with a heterozygous microdeletion in the chromosomal region $7 \mathrm{q} 11.23$ encompassing the elastine gene. The disease is associated with mental retardation and cardiac lesions: SVAS is the most frequent. It is due to an obstructive arteriopathy most prominent at the aortic sinotubular junction.

In the presence of an SVAS, coronary arteries are subject to increased perfusion pressure, leading to dilatation, tortuosity, and accelerated atherosclerosis. True aneurysmal dilatation can happen, but never has an aneurysm of the right coronary artery as big as the one we encountered in association with SVAS and all the clinical and phenotypical signs of Williams syndrome been reported in the available literature. Yilmaz and coworkers ${ }^{1}$ reported 2 cases of adult SVAS associated with aneurysms of the coronary arteries. In both cases surgical intervention was limited to relief of the supravalvular obstruction.

Giant aneurysms of the coronary arteries are rare and can be asymptomatic. They can be discovered as incidental findings during angiography or necroscopy. When symptoms are present, they mimic a space-occupying mediastinal mass with dysphagia, chest pain, and dyspnea. ${ }^{2}$ Differential diagnosis must be performed with acute ischemic heart disease or aortic dissection. Risks of rupture either into one of the cardiac chambers or into the pericardium ${ }^{3}$ are related to their dimensions and are responsible for the onset of symptoms.

Several surgical techniques are reported, such as aneurysmal ligation and vein graft bypass or direct vein graft interposition. In this case we report a direct reimplantation technique to the ascending aorta to ensure that antegrade coronary flow was adopted.

Since the first successful repair in 1956, various surgical techniques to relieve SVAS have been developed. In a recent study from the Boston Children's Hospital, multiple sinus reconstruction resulted in superior hemodynamics and was associated with reduction in both mortality rate and need for reoperation. ${ }^{4}$ In our institution multiple sinus reconstruction, according to the Brom technique, ${ }^{5}$ is the procedure of choice in dealing with SVAS.

\section{Conclusion}

In Williams syndrome a giant aneurysm of the right coronary artery must be considered as part of the natural history of the disease. The cardiac lesions in Williams syndrome are progressive, and for this reason, the case we describe emphasizes the need for close clinical follow-up in this cohort of patients to avoid complications, such as the one reported, which is a surgical challenge with high risks of mortality and morbidity. 


\section{References}

1. Yilmaz AT, Arslan M, Oz̈al E, et al. Coronary artery aneurysm associated with adult supravalvular aortic stenosis. Ann Thorac Surg. 1996;62:1205-7.

2. Mignosa C, Agati S, Ciccarello G, et al. Acute respiratory insufficiency and giant coronary artery aneurysm with fistula. Ann Thorac Surg. 2004;77/5:1823-5.

3. Wan S, Le Clerc JL, Vachiery JL, et al. Cardiac tamponade due to spontaneous rupture of right coronary artery aneurysm. Ann Thorac Surg. 1996;62:575-6.

4. Stamm C, Kreutzer C, Zurakowski D, et al. Forty-one years of surgical experience with congenital supravalvular aortic stenosis. J Thorac Cardiovasc Surg. 1999;118:874-50.

5. Brom AG. Obstruction of the left ventricular outflow tract. In: Khonsari S, editor. Cardiac surgery: safeguards and pitfalls in operative technique. Rockville (MD): Aspen; 1988. p. 276-80. 